\title{
Research on Evaluation Methods for Industrial Product Form Design
}

\author{
LI Fu-xing, SUN Jian, Zhang Yuan-yue \\ Xuzhou institute of technology, Xuzhou 221111, China
}

Keywords: Product Design; Evaluation Method; Shape Modeling; Design Iteration; Scheme Optimization; Design Decision

\begin{abstract}
Design evaluation plays a considerably important role in product development process, and evaluation helps to provide reference for plan improvement or optimization. With the integrative development of different disciplines and improvement of relevant theories, ways to design evaluation methods become diversified, changing from subjective qualitative evaluation to objective quantitative evaluation. From the perspective of industrial design, the typical evaluation methods involved in product design and development process were combed and summarized here, providing reference for further research and bearing certain significance for the construction of systematic product evaluation system and optimization of product design and development process.
\end{abstract}

\section{Introduction}

Design evaluation is the process of judging the value of a design scheme by using the knowledge of design, statistics and other related disciplines in the design practices after weighing comprehensively the various factors affecting the design scheme. As the design and development of industrial products is a continuous iterative process, which makes the evaluation of design schemes in a complex dynamic process, so different indicators of the products in their entire life cycle need to be weighted in a comprehensive way. As an important link in the process of product development, design evaluation can provide an important basis for the improvement or optimization of design schemes through evaluation in different stages of designing. In this article, the author strives to summarize the typical evaluation methods used in the design and development process of products from the perspective of industrial design, to provide a reference for further research.

\section{Qualitative evaluation of product design schemes}

Qualitative evaluation is the process of revealing the characteristic attributes of the object being evaluated in language description to make qualitative conclusions of its value through the valuator's subjective observation and perceptual analysis of the object being evaluated, as shown in Table 1 . 
Table1 Qualitative evaluation of products

\begin{tabular}{c|l}
\hline Evaluation methods & \multicolumn{1}{c}{ Evaluation process } \\
\hline VTs evaluation method & $\begin{array}{l}\text { The product evaluation method of five-element analysis is } \\
\text { proposed in literature. } \\
\text { It is proposed in literature that system analysis be used in the } \\
\text { layout of special vehicle equipment. } \\
\text { It is proposed in literature that the method of standard design be } \\
\text { used for engineering machinery color]. } \\
\text { The evaluation system for products is established in literature } \\
\text { according to users' satisfaction. }\end{array}$ \\
\hline
\end{tabular}

Qualitative evaluation can avoid complicated mathematical calculations, and can go deep into the essence of product design. To solve the problem that product design schemes cannot meet users' needs in some cases, Li Yueen and other people developed a perfect product design evaluation index system through the analysis and study of product design evaluation index at home and abroad. But because the system is not built from the perspective of the users, the evaluation results can not reflect the users' subjective needs objectively. Yao Lan et al. proposed a method for the layout of special vehicle equipment according to the degree of correlation and importance between the evaluation elements. In order to make the evaluation results more convincing, the feasibility verification is conducted through the use of point evaluation method, so as to provide an important method basis for disordered equipment layout. Zhang Chunyan ${ }^{[10]}$ built a product satisfaction evaluation system from the psychological perspective of users, and realized the decision-making evaluation of design schemes. Because the satisfaction degree of the user groups is a multidimensional concept, the study did not formulate corresponding measures for the difference in evaluation results caused by the cognitive bias of the user groups. In addition, users' satisfaction degree for the products will change with the change of surrounding environment and other factors, so in-depth study is needed as how to grasp the points of interest of different groups at different stages[1].

In conclusion, the process of qualitative evaluation is strongly influenced by subjective factors and is difficult to be accurately grasped. As the subject of qualitative evaluation, the valuator's competence will have a great influence on the objectivity of the evaluation results, so the competence of the valuator should be tested and evaluated first. For this issue, relevant scholars proposed the use of sensory evaluation index method and SNR (signal-to-noise ratio) method for evaluation. In addition, in order to give the evaluation process rules to follow and make the evaluation results more convincing, a corresponding evaluation system should be formulated and the evaluation results should be tested for reliability as well.

\section{Quantitative evaluation of product design schemes}

Quantitative evaluation is mainly a process of describing the characteristic attributes of the object being evaluated with numerical value through the use of mathematical formulas and experimental data to make a quantitative judgment of the value of the object being evaluated.

\subsection{An evaluation method based on mathematical models}

\subsubsection{Evaluation of color schemes}

The determination of color scheme for a product is a complex process. To avoid over-reliance on the subjective judgment of the designer and to better solve the uncertainty factors in the process of 
color design, the determination will be made through quantitative evaluation, as shown in Table 2 .

Table 2 Evaluation of color schemes

\begin{tabular}{c|l}
\hline Evaluation method & \multicolumn{1}{c}{ Evaluation process } \\
\hline $\begin{array}{c}\text { Particle swarm optimization } \\
\text { (PSO) }\end{array}$ & $\begin{array}{l}\text { Literature proposes the use of particle swarm optimization for } \\
\text { color scheme evaluation. } \\
\text { Grey Correlation Analysis } \\
\text { Literature proposes that grey correlation analysis be } \\
\text { introduced into color scheme evaluation. } \\
\text { Literature proposes the use of Moon \& Spencer's color } \\
\text { harmony theory in the evaluation of color schemes. } \\
\text { harmony theory color } \\
\text { Literature proposes the evaluation method for color } \\
\text { combination image based on color spatial distribution. } \\
\text { Mathematical models for } \\
\text { Deng's correlation degree }\end{array}$ \\
\hline
\end{tabular}

Ding Man et al. used Particle Swarm Optimization (PSO) to solve the ambiguity in the color image expression of products, and used it as the basis of the method for color scheme optimization. But it is difficult to apply complex calculation process in color design practices. And quantitative processing of color scheme determination will constrain the expression of the final effect of products, because the application of color is mainly a perception issue of the designer. To solve this problem, we can study how to better combine the quantitative processing of color with people's color design perception. Sun Zhixue proposed that users should first mark the color schemes by combing multi-image of the color scheme, and establish the corresponding decision matrix; and then calculate the correlation between each color scheme and the ideal solution by using grey correlation analysis method to realize the optimization of multi-image of color schemes. But users' structural level and cognitive differences will have a great influence on the decision-making result. McClure established a multi-work model of evaluation method for product color combination image based on grey correlation analysis. While taking the color combination images of products in each working mode as an evaluation attribute, the change of the color area of products in each working mode, the subjective images of consumers and other uncertain factors are carefully considered. Therefore, compared with the evaluation process in literature, the evaluation results will be closer to the users' subjective images [2].

Ou Jing et al. proposed the use of Moon \& Spencer's color harmony theory in the optimization of color schemes. The parameters of aesthetic formula are obtained by correlation analysis experiment, and the ideal color scheme is obtained by using the intelligent assistant color matching system. The analysis of color schemes is mainly realized from the psychological perspective in the method, but still lack of research in the quantitative treatment of color design sense. Maison proposed a method of introducing color spatial distribution into product color image evaluation. The color schemes that can satisfy user's preference of image is obtained by introducing influence factors of color space distribution on the basis of Moon \& Spencer's evaluation of aesthetic degree, and take it as an evaluation attribute along with color hue and lightness, and the utility value of each evaluation attribute is obtained by using joint analysis method. As the existence of saltus in the order factors of Moon \& Spencer's aesthetic formula will cause a big error in dealing with the complex color matching, so the evaluation result is inconsistent with user's image perception. Zhang Lin proposes the use of mathematical models for Deng's correlation degree in the evaluation of color schemes. The method is mainly to convert user's subjective fuzzy expression of the product color image into a specific color image evaluation value, so that the image evaluation value of the whole color system can be obtained more accurately and the color design scheme that conforms to the real image of the user can be obtained, too[3]. 


\subsubsection{Evaluation of appearance schemes}

To make the fuzzy factors in the model of product shape be evaluated in a more objective and comprehensive way, quantitative treatment will be used in the evaluation, as shown in Table 3.

Table 3 Evaluation methods of product appearance schemes

\begin{tabular}{c|l}
\hline Evaluation methods & \multicolumn{1}{c}{ Evaluation process } \\
\hline $\begin{array}{c}\text { Fuzzy comprehensive } \\
\text { evaluation }\end{array}$ & $\begin{array}{l}\text { Literature proposes the use of fuzzy comprehensive evaluation for } \\
\text { the optimization of product schemes. } \\
\text { TOPSIS evaluation method }\end{array}$ \\
Naive bayes method & $\begin{array}{l}\text { Literature proposed the use of TOPSIS in the evaluation of design } \\
\text { schemes. } \\
\text { Literature proposed the evaluation method based on naive } \\
\text { bayesian theory for building product availability effect. } \\
\text { Literature proposed design and evaluation methods for product } \\
\text { family. }\end{array}$ \\
\hline
\end{tabular}

In view of the situation that the product shape modeling is difficult to be evaluated objectively because it involves a number of factors, analytic hierarchy process of fuzzy comprehensive evaluation can be used to evaluate the schemes and choose the optimal scheme. In addition, the reasonable determination of the weight value and the evaluation index system will greatly influence the objectivity of the evaluation results. For example, Li Fuxing used fuzzy comprehensive evaluation for the optimization of motorcycle schemes, but used Delphi method for determining the weight value of evaluation index, making the evaluation process with a strong subjectivity. Wang Guiping et al. proposed the use of fuzzy extension AHP method for evaluation. The method is to construct a judgment matrix through the replacement of the number of point values by interval number, and calculate the index weight value of each level. This process is more reasonable than Delphi method. Sarina proposed the use of conjoint variable weight group decision method for evaluation of product schemes. It effectively solved the uncertainty of evaluation index caused by different preferences of individual experts, and realized the balanced evaluation of design schemes.

Pan Ping et al proposed the use of Kansei Engineering for construction of a fuzzy comprehensive evaluation index system. Although the method can guarantee the credibility of the evaluation index system, but it still has a strong subjectivity in the determination of weight value. Su Chen. proposed an evaluation process combining product family design and evaluation method, and constructed corresponding evaluation index system. The selection of evaluation methods in different stages of design can make the evaluation process more targeted, and can realize the best combination of optimal schemes.

In summary, fuzzy comprehensive evaluation uses analytic hierarchy process to construct evaluation index system, which focuses rational evaluation of design process, but the whole process has a low correlation with the users, and the evaluation is mainly based on the opinion of experts. Therefore, in order to make the evaluation results more objective, the determination of weight value should be objective, the construction of the evaluation index system is typical and the structural level of the target group is reasonable[4].

\subsection{The use of experimental evaluation method}

Experimental evaluation method is applied mainly through eye tracking test, electrocardiograph measurement and user's experience. Experimental evaluation method has become an important evaluation method in the process of product development because it can get evaluation feedback on 
products more objectively, as shown in Table 4.

Table 4 The use of experimental evaluation method

\begin{tabular}{c|l}
\hline Experimental methods & \multicolumn{1}{c}{ Experimental evaluation process } \\
\hline Eye tracking test & $\begin{array}{l}\text { Literature proposed the use of eye tracking test for the } \\
\text { evaluation of product appearance. } \\
\text { Literature proposed the use of electromyography measurement } \\
\text { and Borg scale to establish a performance evaluation model of } \\
\text { massage chair. } \\
\text { Literature proposed the use of EEG measurement to study the } \\
\text { measurement } \\
\text { rules of massage comfort and EEG change. } \\
\text { Literature proposed the use of body pressure distribution test to } \\
\text { evaluate the comfort of chairs. }\end{array}$ \\
Body pressure \\
distribution test \\
Experiential test
\end{tabular}

The experimental evaluation data of the product form can be obtained by the external manifestation of the valuators' subjective psychological activity. Hu Weifeng et al. used the eye tracking technique to evaluate the shape schemes of fracturing vehicles from the concentration of the fixation point and the distribution of the hot area, and summed up the methods and rules guiding the design of product shape. Eye tracking technology can get feedback on the shape of products in a more intuitive way, so as to make the design more targeted, as shown in Figure 1. But the experiment is only focused on the analysis of concentration of fixation points and the distribution of the hot spots, but there is a lack of research on the hot spots, the line of sight scanning, the area of interest and so on. In addition, there are differences in different groups' area of concern for the product, so it is necessary to carry out targeted research on the groups closely related to the product. Li Yun et al. proposed the method to build a model of product modeling based on a number of eye movement data, and verified that the model can effectively reflect users' comprehensive evaluation of each scheme, thus effectively overcome the limitation of single eye movement evaluation data in literature [5].

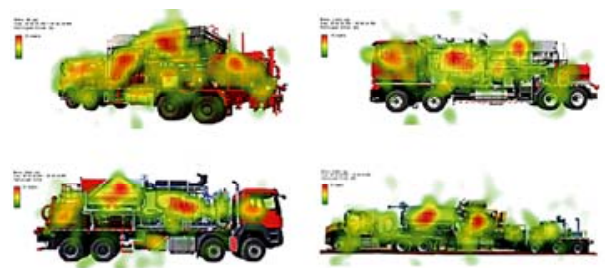

Figure 1 Eye tracking test for product shape modeling

Physiological index data are widely used as the main basis for evaluating the rationality of a product. For example, Yang Zhongliang et al have studied the performance of massage chairs with the method of EMG measurement. The purpose is to verify the validity of the experimental method by comparing the effectiveness of massage and sitting still for alleviating fatigue. Diego evaluates the performance of massage chairs with EEG measurement, and deals with experimental data by using eye movement correction, filtering and other methods. He found out through experimental measurement that the same massage mode used in different part will produce different feeling. This can provide a basis for setting up the massage mode of a massage armchair according to the part of body. Liu Weisong evaluated the comfortableness of chairs through body pressure distribution test and learned that individual difference has great influence on the comfortableness of the seat surface, thus provided a foundation for designing schemes for specific users. 
Experiential testing is used as an important evaluation method. For example, Noto $\mathrm{Y}$ has evaluated products through user's usability experience. The evaluation process can objectively reflect the psychological state in operation, which can be considered the best way to evaluate the product designs. But the value of evaluation information feedback mainly depends on the user's level of knowledge, and cognitive differences may lead to different evaluation results. Regarding this, it is necessary to formulate corresponding experiential test evaluation criteria[6].

In summary, further analysis and research needs to be made as to whether the feedback on the evaluation of comfortableness in various experimental methods is consistent, and whether there is any difference between the experimental data and subjective evaluation.

\subsection{Evaluation method based on software platform}

Comfortableness evaluation of a product based on software platform and related theory, and product design iteration according to the result of evaluation has been widely used in product design and development process, as shown in Table 5.

Table 5 Evaluation method based on software platform

\begin{tabular}{c|l}
\hline $\begin{array}{c}\text { Software } \\
\text { platform }\end{array}$ & \multicolumn{1}{c}{ Specific evaluation process } \\
\hline ADAMS- & $\begin{array}{l}\text { Xiang Zhongxia et al. proposed the evaluation method for bicycle man - } \\
\text { machine system based on human biomechanics and ADAMS - Life Mod } \\
\text { Life Mod }\end{array}$ \\
SATIA & $\begin{array}{l}\text { Li Fuxing proposed the method for evaluation of the comfortableness of } \\
\text { vehicle repair operations based on CATIA software. } \\
\text { Zhang Lei et al. studied the relationship between shape modeling and } \\
\text { comfortable of products based on Pro / E and ANSYS softwares. } \\
\text { Zhang Yong evaluated the comfortableness of rotating seats on high-speed } \\
\text { trains based on JACK software platform. } \\
\text { Andreas proposed a method of evaluating drivers' comfortableness in } \\
\text { operation based on RAMSIS software. }\end{array}$ \\
JACK & \multicolumn{1}{c}{ RAMSIS }
\end{tabular}

Literature evaluated the comfortableness of vehicle repair operations based on CATIA software, during which man-machine coupling can be set up according to simulation needs. But in the process of evaluation based on the software platform, man-machine coupling will be constrained due to body size. As the software platform developed by European and American countries lacks the database of Chinese human body size, there will be deviation from the objective reality in the evaluation of man-machine coupling. Therefore, researchers have proposed corresponding correction and improvement methods. For instance, Morten proposed the use of Human Modeling module of UG software platform and Grip secondary development tool in rebuilding the evaluation system, which is mainly to put the data provided by GB10000-1988, after correction and improvement, into the software platform to realize man-machine matching coupling; Li Fuxing et al proposed the method of choosing first the human body models with similar size from the software platform, and then correcting them in the human body model editing module according to the data provided by GB 10000-1988, but the method may result in a disproportionate ratio between various parts of the human body model.

The above research shows that in the process of human-machine coupling evaluation based on the software platform, the evaluation of comfortableness is only made from physiological angle, and ignored psychological factors; the use of products is a dynamic continuous process, selecting only a few representative performance postures for comfortableness evaluation lacks persuasiveness for extensive evaluation results. In addition, human joint comfort level has a wider scope of 
application than human body structure size in evaluation [7].

\section{Comprehensive evaluation of product design schemes}

In view of the complexity in the process of product design and development, it is difficult to conduct objective evaluation by using a single evaluation method. Regarding this, qualitative evaluation of products should be combined with quantitative evaluation to realize comprehensive evaluation of design schemes, as shown in Table 6.

Table 6 Comprehensive evaluation method of product shape modeling schemes

\begin{tabular}{|c|c|}
\hline Evaluation method & Specific evaluation process \\
\hline Cognitive & Literature studied the method for improving the operation efficiency \\
\hline psychological & of machine tool's man-machine interface according to the theory of \\
\hline evaluation & nitive psychology \\
\hline $\begin{array}{l}\text { Principal component } \\
\text { analysis (PCA) }\end{array}$ & $\begin{array}{l}\text { Literature proposed the use of principal component analysis to } \\
\text { evaluate the appearance of products" }\end{array}$ \\
\hline $\begin{array}{l}\text { Support vector } \\
\text { regression }\end{array}$ & Literature proposed the method for evaluating the comfort level of \\
\hline $\begin{array}{l}\text { Kandall coefficient of } \\
\text { concordance }\end{array}$ & $\begin{array}{l}\text { driving seat based on support vector regression. } \\
\text { Literature studied the method for evaluating the perceptual images of } \\
\text { products in different states through the use of Kandall coefficient of } \\
\text { concordance. }\end{array}$ \\
\hline $\begin{array}{l}\text { Gene expression } \\
\text { programming }\end{array}$ & $\begin{array}{l}\text { Literature proposed the method for subjective evaluation of } \\
\text { comfortableness through gene expression programming. }\end{array}$ \\
\hline
\end{tabular}

To make the evaluation result of product design schemes more objective, it should be verified by combing the corresponding experimental method. For instance, Gu Yi et al conducted a qualitative analysis of the degree of influence by various factors on the operating efficiency in man-machine interface through predicted method, and conducted quantitative measurement of each factor's degree of influence on the efficiency of interface through simulation experiments. The application of principles of psychology in the evaluation process expands the degree of sensibility of the evaluation system and will be more in line with the user's psychological perception. Yang Cheng et al. emphasized the construction of the product evaluation index system and the verification of evaluation results. They first broadly selected the evaluating indicators through survey and study; and then screened the evaluating indicators through evaluation and test to improve their value; finally they built the evaluation matrix based on the value of subjective and objective index to get their principal components and contribution rates, and verified the results through experiments [8] .

Niels proposed the use of evaluation scale and Kandall coefficient of concordance to evaluate the perceptual image effect of a product in three-dimensional and plane states respectively, in order to grasp the effect of product shape design in a comprehensive way. Whether the evaluation process is interrelated with the methods proposed in literature requires an in-depth analysis and study. Shi Qingchun et al. proposed a quantitative product design method based on users' objective cognition to study the degree of matching between the appearance of products and users' emotional needs. They screened out style image vocabulary and product samples to build a mapping relationship between typical perceptual image vocabulary and key modeling characteristics of a product; and then conducted eye tracking test for the image scale of feature element, and designed the shape of the product according to the test results. The application of SD method and eye tracking device gave an objective basis to the entire design process, and will serve as a more valuable reference for product shape design. 


\section{Conclusion}

Researchers have done a lot of studies on the methods for evaluation of the shape design schemes of industrial products, and have achieved fruitful results. The following conclusions have been obtained through the analysis of relevant literatures:

(1) Qualitative evaluation can decide the product scheme, but the decision-making process is greatly influenced by subjective factors, so the differences in cognitive ability of valuators will lead to different evaluation results. In addition, the short of support of objective data in the evaluation process makes the evaluation results lack of credibility, and appropriate methods are needed for validation. Therefore, qualitative evaluation is more suitable for exploratory research process;

(2) Accurate calculation of the evaluation process can be realized based on mathematical models, but for industrial designers, complex calculation process is hard to be applied in design practice, and subjective feelings and other factors can not be objectively reflected. So it is more focused on the research in theoretical level; objective indicators of data can be obtained through the use of experimental methods, software platform and other evaluation methods, which has an important reference value for the design and development of products, but users' subjective feeling cannot be objectively reflected, either. Therefore, users' subjective feeling can be further studied on the basis of quantitative evaluation;

(3) Comprehensive evaluation is the combination of qualitative and quantitative evaluation, which can realize an overall evaluation of product design schemes, but there are a number of methods for evaluating the various factors of products, and each method has some constraint. To solve this issue, the evaluation methods for each factor of a product can be integrated after improvement to build a systematic product evaluation system.

\section{References}

[1] Hartson, Terence S Andre.Criteria for evaluating usability evaluation methods[J].International Journal of Human Computer Interaction,2003,15 (1) :145-181.

[2] McClure, WUFG . A design decision-making support for customized product color combination [J].Computers in Industry,2007,58(6):504-518.

[3] Maison D, Greenwald. User-oriented design for the optimal combination on product[J].International Journal of Production Ergonomics,2006,100(2):253-267.

[4] Diego, Field T. Massage therapy of Moderate and light pressure and vibrator effects on EEG and heart rate[J].International Journal of Neuroscience,2004,114(1):31-44.

[5] Noto Y, Kudo M and Hirota K. Back massage therapy pro motes psychological relaxation and an increase in salivary chronograph a release [J]. Japanese Society of Anesthesiologists,2010,24(6):955-958.

[6] Andreas.The influence of design aesthetics in usability testing:effects on user performance and perceived Applied Ergonomics,2010,41 (3):403-410.

[7] Morten.How do usability professionals construe usability [J].International Journal of Human Computer Studies,2012, 70 (1) :26-42.

[8] Niels, M Moes.Variation in sitting pressure distribution and location of the points of maximum pressure with rota -tion of the pelvis, gender and body characteristics[J]. Ergonomics,2007,50 (4):536-561. 